5. Ruengwanichayakun P, Gambarotti M, Frisoni T, et al. Parosteal osteosarcoma: a monocentric retrospective analysis of 195 patients. Hum Pathol 2019; 91: 11-8.

6. Yoshida A, Ushiku T, Motoi T, et al. Immunohistochemical analysis of MDM2 and CDK4 distinguishes low-grade osteosarcoma from benign mimics. Mod Pathol 2010; 23: 1279-88.

7. Dujardin F, Binh MB, Bouvier C, et al. MDM2 and CDK4 immunohistochemistry is a valuable tool in the differential diagnosis of lowgrade osteosarcomas and other primary fibro-osseous lesions of the bone. Mod Pathol 2011; 24: 624-37.

8. Duhamel LAE, Ye H, Halai D, et al. Frequency of mouse double minute 2 (MDM2) and mouse double minute 4 (MDM4) amplification in parosteal and conventional osteosarcoma subtypes. Histopathology 2012; 60: 357-9.

9. Grimer RJ, Carter SR, Spooner D, Sneath RS. Diagnosing musculoskeletal tumours Sarcoma 2001; 5: 89-94.

10. Yoshida A, Ushiku T, Motoi T, et al. MDM2 and CDK4 immunohistochemical coexpression in high-grade osteosarcoma: correlation with a dedifferentiated subtype. Am J Surg Pathol 2012; 36: 423-31.

11. Wunder JS, Eppert K, Burrow SR, et al. Co-amplification and overexpression of CDK4, SAS and MDM2 occurs frequently in human parosteal osteosarcomas. Oncogene 1999; 18: 783-8.

12. Dorfman HD, Ishida T, Tsuneyoshi M. Exophytic variant of fibrous dysplasia (fibrous dysplasia protuberans). Hum Pathol 1994; 25: 1234 -

DOI: https://doi.org/10.1016/j.pathol.2020.08.018

\section{A challenging case of a poorly differentiated small cell variant of synovial sarcoma with unusual immunophenotype}

Sir,

Diagnosis of a small cell variant of a poorly differentiated synovial sarcoma (PDSS) can be very challenging given the rarity of this subtype of synovial sarcoma ${ }^{1}$ and the very broad pathological differential diagnosis with other 'small blue round cell tumours', including various small round cell sarcomas, (small cell) carcinoma, lymphoblastic lymphoma and (small cell) melanoma. Recently, we have encountered a challenging case of a poorly differentiated small cell variant of a synovial sarcoma with an unusual immunophenotype.

A 35-year-old woman, without a history of trauma, presented with a soft tissue mass of the right subclavicular region. On clinical examination, the swelling was hard to palpate and measured approximately $6 \times 6 \mathrm{~cm}$. Magnetic resonance imaging (MRI) of the mass showed a lobulated, cystic soft tissue tumour with calcifications. After multidisciplinary discussion, an incision biopsy was performed. Microscopy showed a highly cellular, poorly differentiated malignant tumour composed of compact sheets of relatively uniform rounded cells with a high nuclear to cytoplasmic ratio (Fig. 1A). The cells had scant cytoplasm with indistinct borders ('small blue round cell' morphology) and contained vesicular nuclei with prominent nucleoli, demonstrating nuclear overlap (Fig. 1C). Brisk mitotic activity was observed (over 30 mitoses/10 high power fields). Very focally, an increase of myxoid stroma with ectatic (staghorn-like) vessels could be seen (Fig. 1B,D). Tumour necrosis and calcifications were absent. Immunohistochemistry showed diffuse, membranous staining for CD99 (Fig. 2A), together with strong nuclear transducing-like enhancer of split 1 (TLE1) reactivity of tumour cells (Fig. 2B). Patchy expression for epithelial membrane antigen (EMA) (Fig. 2C), cytokeratin AE1/AE3 (Fig. 2D), BCOR (Fig. 2E) and desmin (Fig. 2F) was observed. No aberrant immunoreactivity was seen for SOX10, S100, CD45, terminal deoxynucleotide transferase (TdT), myeloperoxidase (MPO), insulinoma associated protein 1 (INSM1), MyoD1, myogenin (MYF4), NKX2.2,

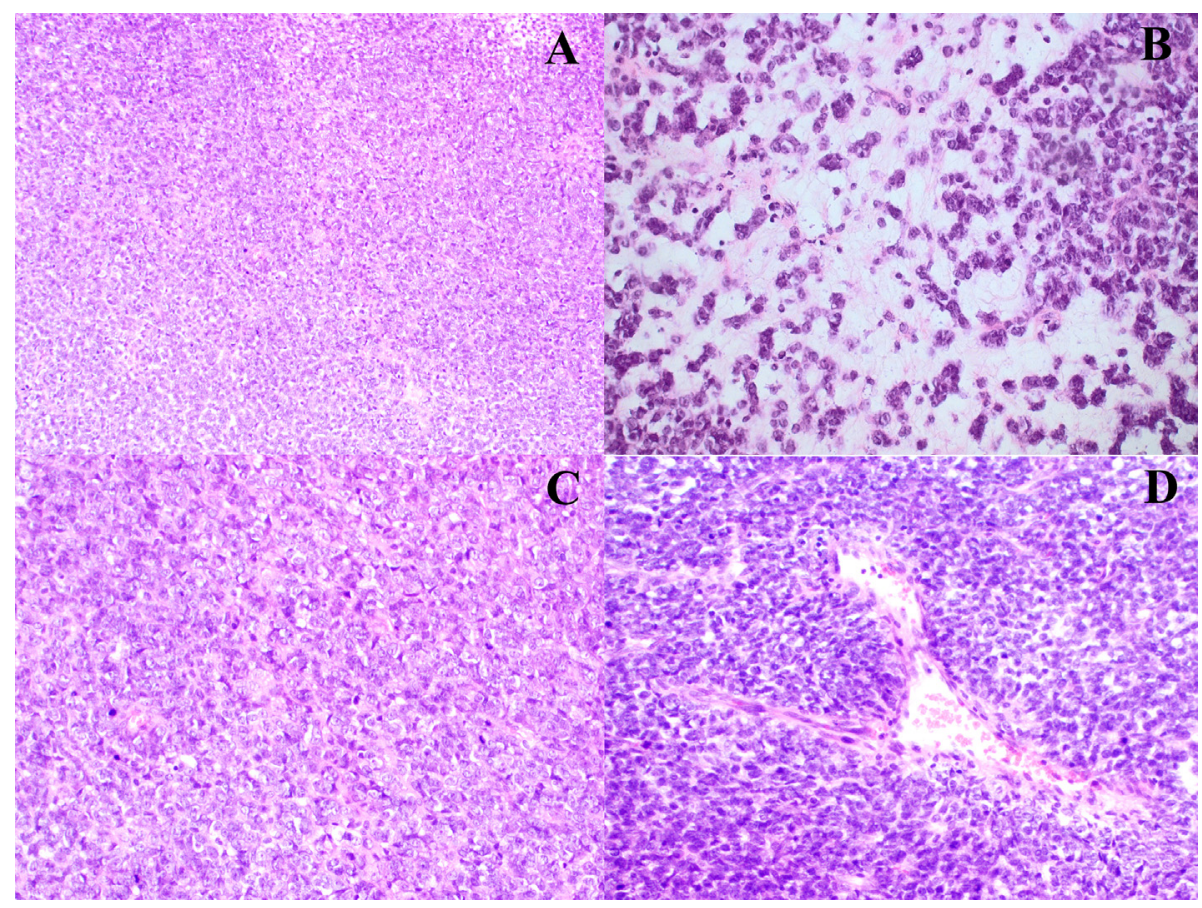

Fig. 1 (A) Highly cellular, malignant tumour composed of compact sheets of poorly differentiated rounded cells (H\&E). (B) Area with an increased myxoid stroma (H\&E). (C) Poorly differentiated rounded cells with a high nuclear to cytoplasmic ratio, vesicular nuclei with nucleoli and brisk mitotic activity (H\&E). (D) Presence of an ectatic (staghorn-like) vessel (H\&E). 


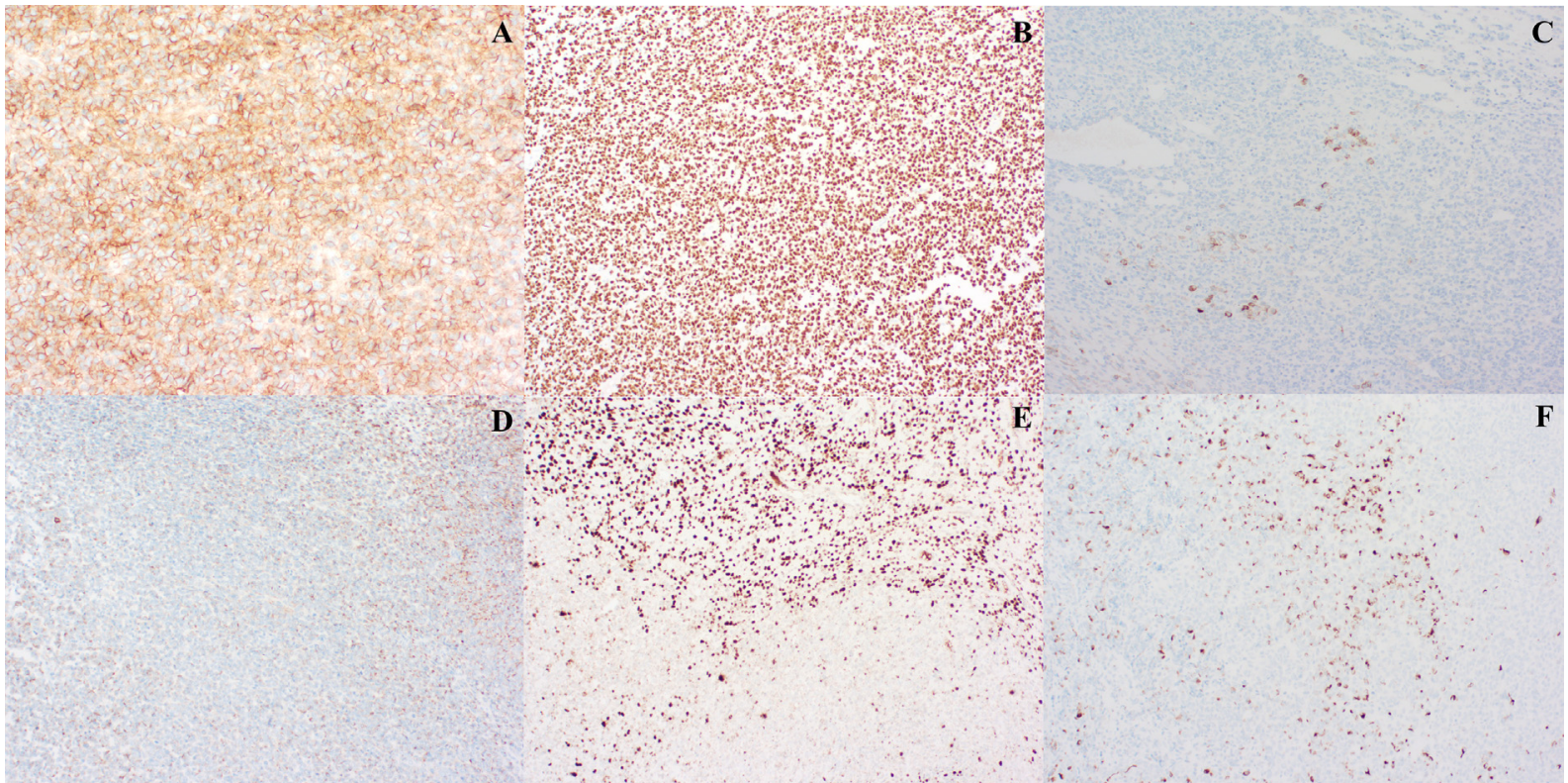

Fig. 2 (A) Tumour cells showing diffuse, membranous staining for CD99. (B) Strong nuclear TLE1 reactivity of the tumour cells. (C) Patchy expression of the tumour cells for EMA, (D) cytokeratin AE1/AE3, (E) BCOR, and (F) desmin.

special AT rich sequence binding protein (SATB2), Wilms' tumour 1 (WT1), ETS translocation variant 4 (ETV4), ERG, NKX3.1, pan-tropomyosine receptor kinase (pan-Trk) and mucin 4 (MUC4). Nuclear SMARCB1 (INI-1), SMARCA2 and SMARCA4 expression was retained in the tumour. Fluorescence in situ hybridisation (FISH) was performed using break apart probes for SS18 (SYT), EWSRI and FUS, which revealed $S S 18$ gene rearrangement and did not show rearrangement of FUS or EWSR1. Based on the poorly differentiated 'small blue round cell' morphology, the immunohistochemical profile (membranous staining for CD99, diffuse nuclear TLE1 positivity and patchy expression of cytokeratin AE1/AE3 and EMA) and molecular findings (rearrangement of the SS18 gene) the diagnosis of a poorly differentiated small cell variant of a synovial sarcoma was made.

Ewing sarcoma (ES), being the prototypic round cell sarcoma, could be considered in this case due to the strong membranous CD99 staining. However, the relatively old age of the patient, the strong diffuse expression for TLE1 and the negative staining for NKX2.2 (which is a highly sensitive marker for ES), ${ }^{2}$ argue against a diagnosis of a classical ES. In addition, no EWSRI or FUS gene rearrangements could be demonstrated in this case. 'Ewinglike' sarcomas, including $B C O R$ rearranged sarcoma and $C I C$ rearranged sarcoma, should also be included in the differential diagnosis of PDSS. Moreover, BCOR rearranged sarcoma expresses TLE1 in most cases, which can be a major diagnostic pitfall in the differential diagnosis with synovial sarcoma. ${ }^{3}$ BCOR overexpression is a highly sensitive marker for $B C O R$ rearranged sarcomas, typically showing a strong and diffuse nuclear positivity (in contrast to the only patchy weak to moderate BCOR immunoreactivity seen in this case). ${ }^{4}$ However, BCOR expression is not a specific marker for $B C O R$ rearranged sarcomas and is often observed in synovial sarcoma. Most cases of $B C O R$ rearranged sarcomas also express SATB2 (no SATB2 expression was observed in current case). ${ }^{4}$ Some morphological features seen in this case (in particular the relatively uniform cytomorphology with vesicular chromatin and visible nucleoli, the brisk mitotic activity and the focal myxoid stromal changes) are also described in CIC rearranged sarcoma. ${ }^{5}$ Moreover, $C I C$ rearranged sarcomas can express CD99 in a diffuse and membranous pattern $(20 \%$ of cases) and are typically negative for NKX $2.2 .^{5}$ On the other hand, the current case did not express WT1 and ETV4, both being very sensitive markers for CIC rearranged sarcoma. ${ }^{6}$ Interestingly in 2018, Alholle et al. identified a novel recurrent gene fusion, CRTC1-SS18, in two undifferentiated round cell sarcomas both located in the soft tissue of the lower extremities of young adults with an 'Ewing-like' morphology and strong CD99 immunopositivity. However, these exceptional cases showed a morphology (tumour nuclei with stippled chromatin and a prominent desmoplastic stroma reminiscent of desmoplastic small round cell tumour) and immunoprofile (the tumour cells did not stain for cytokeratin AE1/AE3, EMA, desmin, BCOR or TLE1) clearly different from our current case. Alternatively, a SS18-POUSF1 fusion was very recently reported in two undifferentiated round cell sarcomas located intra-abdominal and within the abdominal wall in young patients. ${ }^{8}$ The morphology (tumour nuclei with fine chromatin and the presence of a desmoplastic fibrotic stroma) and the immunohistochemistry (both tumours showed S100 expression and only focal weak TLE1 reactivity was observed in one of the two cases) are also not comparable to those described in this case. Unforeseen in our case was the patchy desmin expression, which is an exceptional finding in synovial sarcoma. ${ }^{1,9,10}$ Given the 'unexpected' desmin expression, additional myogenin and MyoD1 stainings (both were negative) were performed to exclude a rhabdomyosarcoma. Interestingly, polyphenotypic differentiation (including desmin expression) is recently described in 'Ewing-like' round cell sarcomas with 
EWSR1-PATZ1 gene fusion. ${ }^{11,12}$ No EWSR1 gene rearrangement could be demonstrated using FISH, whereby this differential diagnosis could also be excluded.

In conclusion, we present a case of an unusual small cell variant of a PDSS, which is a histological mimic of other 'small blue round cell tumours'. The 'unforeseen' positivity for desmin made this case even more challenging. The morphology and the immunohistochemical profile (membranous staining for CD99, diffuse nuclear TLE1 positivity and patchy expression of cytokeratin AE1/AE3 and EMA) raised awareness for the diagnosis of a PDSS in this case. Molecular testing, confirming SS18 (SYT) gene rearrangement, remains the gold standard for the diagnosis of this rare morphological subtype of synovial sarcoma.

Conflicts of interest and sources of funding: The authors state that there are no conflicts of interest to disclose.

\section{Fleur Cordier $^{1}$, Lore Lapeire ${ }^{2,3}$, Gwen Sys ${ }^{3,4}$, Jo Van Dorpe $^{1,3}$, David Creytens ${ }^{1,3}$}

${ }^{1}$ Department of Pathology, Ghent University Hospital, Ghent University, Ghent, Belgium; ${ }^{2}$ Department of Medical Oncology, Ghent University Hospital, Ghent University, Ghent, Belgium; ${ }^{3}$ CRIG, Cancer Research Institute Ghent, Ghent University Hospital, Ghent University, Ghent, Belgium; ${ }^{4}$ Department of Orthopedics and Traumatology, Ghent University Hospital, Ghent University, Ghent, Belgium

Contact Prof David Creytens.

E-mail: david.creytens@uzgent.be

1. Suurmeijer AJH, Ladanyi M, Nielsen TO. Synovial sarcoma. In: WHO Classification of Tumours Editorial Board. WHO Classification of Tumours: soft Tissue and Bone Tumours. Lyon: IARC Press, 2020.

2. Hung YP, Fletcher CDM, Hornick JL. Evaluation of NKX2-2 expression in round cell sarcomas and other tumors with EWSR1 rearrangement: imperfect specificity for Ewing sarcoma. Mod Pathol 2016; 29: 370-80.

3. Li WS, Liao IC, Wen MC, Lan HH, Yu SC, Huang HY. BCOR-CNB3positive soft tissue sarcoma with round-cell and spindle-cell histology: a series of four cases highlighting the pitfall of mimicking poorly differentiated synovial sarcoma. Histopathology 2016; 69: 792-801.

4. Kao YC, Owosho AA, Sung YS, et al. BCOR-CCNB3-fusion positive sarcomas: a clinicopathologic and molecular analysis of 36 cases with comparison to morphologic spectrum and clinical behavior of other round cell sarcomas. Am J Surg Pathol 2018; 42: 604-15.

5. Antonescu CR, Owosho AA, Zhang L, et al. Sarcomas with CICrearrangements are a distinct pathologic entity with aggressive outcome: a clinicopathologic and molecular study of 115 cases. Am J Surg Pathol 2017; 41: 941-9.

6. Hung YP, Fletcher CD, Hornick JL. Evaluation of ET4 and WT1 expression in CIC-rearranged sarcomas and histologic mimics. Mod Pathol 2016; 29: 1324-34.

7. Alholle A, Karanian M, Brini AT, et al. Genetic analyses of undifferentiated small round cell sarcoma identifies a novel sarcoma subtype with a recurrent CRTC1-SS18 gene fusion. J Pathol 2018; 245: 18696.

8. Antonescu CR, Agaram NP, Sung YS, Zhang L, Dickson BC. Undifferentiated round cell sarcomas with novel SS18-POU5F1 fusions. Genes Chromosomes Cancer 2020; 59: 620-6.

9. Pelmus M, Guillou L, Hostein I, Sierankowski G, Lussan C, Coindre JM. Monophasic fibrous and poorly differentiated synovial sarcoma: immunohistochemical reassessment of $60 \mathrm{t}(\mathrm{X} ; 18)$ (SYT-SSX)positive cases. Am J Surg Pathol 2002; 26: 1434-40.

10. Changchien YC, Katalin U, Filinger J, et al. Case report: a challenging case of metastatic intra-abdominal synovial sarcoma with unusual immunophenotype and its differential diagnosis. Case Rep Pathol 2012; 2012: 78683.
11. Chougule A, Taylor M, Nardi V, et al. Spindle and round cell sarcoma with EWSR1-PATZ1 gene fusion: a sarcoma with polyphenotypic differentiation. Am J Surg Pathol 2019; 43: 220-8.

12. Bridge JA, Sumegi J, Druta M, et al. Clinical, pathological and genomic features of EWSR1-PATZ1 fusion sarcoma. Mod Pathol 2019; 32: $1593-604$.

DOI: https://doi.org/10.1016/j.pathol.2020.08.028

\section{Malignant transformation of liposclerosing myxofibrous tumour}

Sir,

Liposclerosing myxofibrous tumour (LSMFT) is a benign fibro-osseous bone lesion that was first described by Ragsdale and Sweet in $1986 .{ }^{1}$ LSMFT is a comparatively rare bone lesion, and it is thought to be related to fibrous dysplasia (FD) because of similarities in their histological and radiographic features. A recent molecular study provided additional evidence for the relationship between LSMFT and FD: an activating point mutation in the alpha subunit of a $G$ protein (GNAS) was identified in some LSMFT samples, and the same mutation is present in nearly all cases of polyostotic and monostotic FD. ${ }^{2-5}$ Only a few cases of spontaneous 'malignant transformation' (MT) of LSMFT have been reported. ${ }^{1,2,6,7}$ To the best of our knowledge, this is the first reported case of MT in LSMFT in association with presumed polyostotic FD.

This study was approved by the institutional review board (IRB) of our hospital (No. UC18SESI0111). Informed consent was obtained from the patient for publication of this case.

A 75-year-old female patient with left knee joint pain was referred to our hospital. Plain radiographs of both knees revealed osteoarthritis of the left knee joint (Fig. 1A). In addition, multiple osteolytic and sclerotic lesions were incidentally found in the right proximal tibia and distal femur (Fig. 1A,B, arrows). These lesions demonstrated sclerotic rims, internal septations, and calcifications. Plain radiograph of the pelvis showed lobular lesions with ground glass opacity in the bilateral femur necks to the proximal metaphysis (Fig. 1C, arrow). An osteolytic, sclerotic lesion was also detected in the skull occiput. Magnetic resonance images of the right proximal femur diaphysis and metaphysis revealed a $10 \times 3 \mathrm{~cm}$ lesion without evidence of cortical breakage or expansion. This lesion showed heterogeneously low signal intensity on $\mathrm{T} 1$ weighted image (WI) and heterogeneously high signal on T2WI. Another lesion in the left femur neck showed high signal intensity on T2WI, indicative of avascular necrosis (AVN). Based on these imaging findings, the radiological diagnoses of the patient were the following: (1) presumed polyostotic FD involving right distal femur, right proximal tibia, and skull; (2) LSMFT of the intertrochanteric area of right femur; (3) osteoarthritis of the left knee joint; and (4) AVN of the left femur head. A total knee replacement arthroplasty was performed for the left knee joint osteoarthritis. Radiographic follow-up was recommended for the other lesions (presumed polyostotic FD, AVN, and LSMFT). After 2.3 years, the follow-up radiograph of the left femur neck lesion showed progression (Fig. 1D, black arrow). The patient 